\title{
Developing consistency in teacher judgement formation through online meeting centres
}

\author{
Lenore Adie \\ Faculty of Education \\ Queensland University of Technology, AUSTRALIA \\ lenore.adie@qut.edu.au
}

\begin{abstract}
This paper investigates the complex interactions that occur as teachers meet online to justify and negotiate their assessment judgements of student work across relatively large and geographically dispersed populations. Drawing from sociocultural theories of learning and technology, the technology is positioned as playing a role in either supporting or hindering teachers reaching a common understanding of assessment standards. Meeting transcripts and interviews with the teachers have been qualitatively analysed in terms of the interactions that occurred and teachers' perceptions of these interactions. While online meetings offer a partial solution to address the current demands of assessment in education, they also present new challenges as teachers meet, in an unfamiliar environment, to discuss student work.
\end{abstract}

Keywords

social moderation, assessment, online meeting centres, teacher judgement

\section{Introduction}

Information and communication technology (ICT) -mediated moderation is a new and unexplored domain for classroom teachers to meet and discuss their understanding of what denotes quality in student work. Advancements in communication technologies offer an exciting development for connecting teachers. While there has been considerable work undertaken in relation to online assessment practices in the tertiary sector (for example, Salmon, 2003), this work mostly relates to asynchronous e-moderation and to e-learning environments. The technical difficulties experienced by participants in both modes of operating (synchronous and a-synchronous) may be similar in terms of sending work samples, but synchronous online moderation as presented in this paper adds another complication in terms of spoken dialogue between participants. Little is known of the effect of meeting in a synchronous online environment to moderate student work for classroom teachers and in the course of the discussion to develop common understandings of pre-determined standards.

This paper is based on an ongoing research project that is investigating the formation of a common understanding of defined standards when teachers meet to moderate student work within a synchronous online environment. The paper is focusing on the process of online social moderation, and the factors that may support or hinder teachers in their judgement role. Specifically, in this paper the link between the technology and the meeting participants is examined.

The study draws upon the current implementation of a new curriculum, assessment and reporting framework in the Australian state of Queensland. The Queensland Curriculum, Assessment and Reporting (QCAR) framework ( Department of Education Training and the Arts, 2005) focuses on the middle years of schooling and involves the establishment of essential learnings, defined 
standards and a common reporting system that aims to promote consistency of teacher judgement. The QCAR framework involves students in Years 4, 6 and 9 completing comparable assessment tasks in the stated Essential Learnings (Queensland Studies Authority, 2007). The quality of student work is then judged using defined A to E standards. The assumption is that an 'A' standard awarded in the far north of Queensland is comparable to the 'A' standard awarded in metropolitan Brisbane in the south-eastern corner of the state (Department of the Premier and Cabinet, 2005). For this consistency to be realised, Queensland teachers need to develop a shared understanding of the standards and the assessment concepts used in a standards-based system.

It is envisioned that one way of developing and achieving consistency in the understanding of the standards is through the participation by teachers in moderation meetings. Social moderation is defined as "a process for developing consistency or comparability of assessment judgements across different assessors, programs and schools" (Maxwell, 2002, p. 1). During these meetings, teachers gather to negotiate and discuss their understandings of the standards in order to reach agreement on the level of achievement reflected within samples of students' work. In such an environment teachers justify their judgements of students' work and in the process expose their tacit understandings and beliefs, or their "internalized reflective knowledge" (Fehring, 1998, p. 12) regarding what they consider as quality in student work. Research (Davidson, 1999; Ingvarson, 1990; Klenowski, 2007; Malone, Long, \& De Lucchi, 2004) has provided some evidence of the role of social moderation meetings to support the development of shared understandings of standards and even the formation of an assessment 'community of practice' within schools or districts. Problematic for this discussion is the range of influence of such meetings within educational systems that embrace standards-based assessment yet require that consistency of teacher judgement is established and maintained.

While a moderation process has been in operation in Queensland since 1972 for Years 11 and 12 in relation to the Queensland senior syllabus (Queensland Studies Authority, 2005), this procedure has not been organized for the middle years of schooling. The result has meant a lack of consistency and continuity, and a lack of knowledge of the assessment and reporting practices used in these years, with no formally endorsed assessment and reporting framework until now. The introduction of this standards-driven curriculum, assessment and reporting framework has meant a new way of working for middle school teachers. However, organising and conducting social moderation meetings for the middle years of schooling is a fiscally and logistically prohibitive process due to the large numbers of schools catering to the middle years (approximately 1700 schools across Queensland), and the isolation and vast distances between schools particularly in many rural areas of Queensland. Online moderation meetings have been proposed as a way to meet systemic calls for consistency of teacher judgements while facilitating the development of an assessment 'community of practice' (Lave \& Wenger, 1991) amongst diversely situated teachers.

This study involved teachers gathering together using the $\mathrm{WebEx}^{\odot}$ online meeting centre [see http://www.webex.com.au]. WebEx ${ }^{\Theta}$ allows for audio, video and text to be incorporated in meetings through the sharing of documents, applications or desktops. Participants are invited through email and communicate in the meeting through their telephone link up while interacting with the materials online. Features like the hands-up icon allow participants the opportunity to have their opinions heard. Participants meet in real time to view or annotate student work samples using highlighters, text or pointers. Figure 1 shows a screen shot of one of the online moderation meetings in progress. The author/researcher acted as organiser and facilitator of these meetings. This involved inviting the teachers to participate in the meetings, uploading student tasks, assisting teachers with technological difficulties, and inviting participation throughout the meeting. It did not involve participation in the discussions about the moderation of student work. 


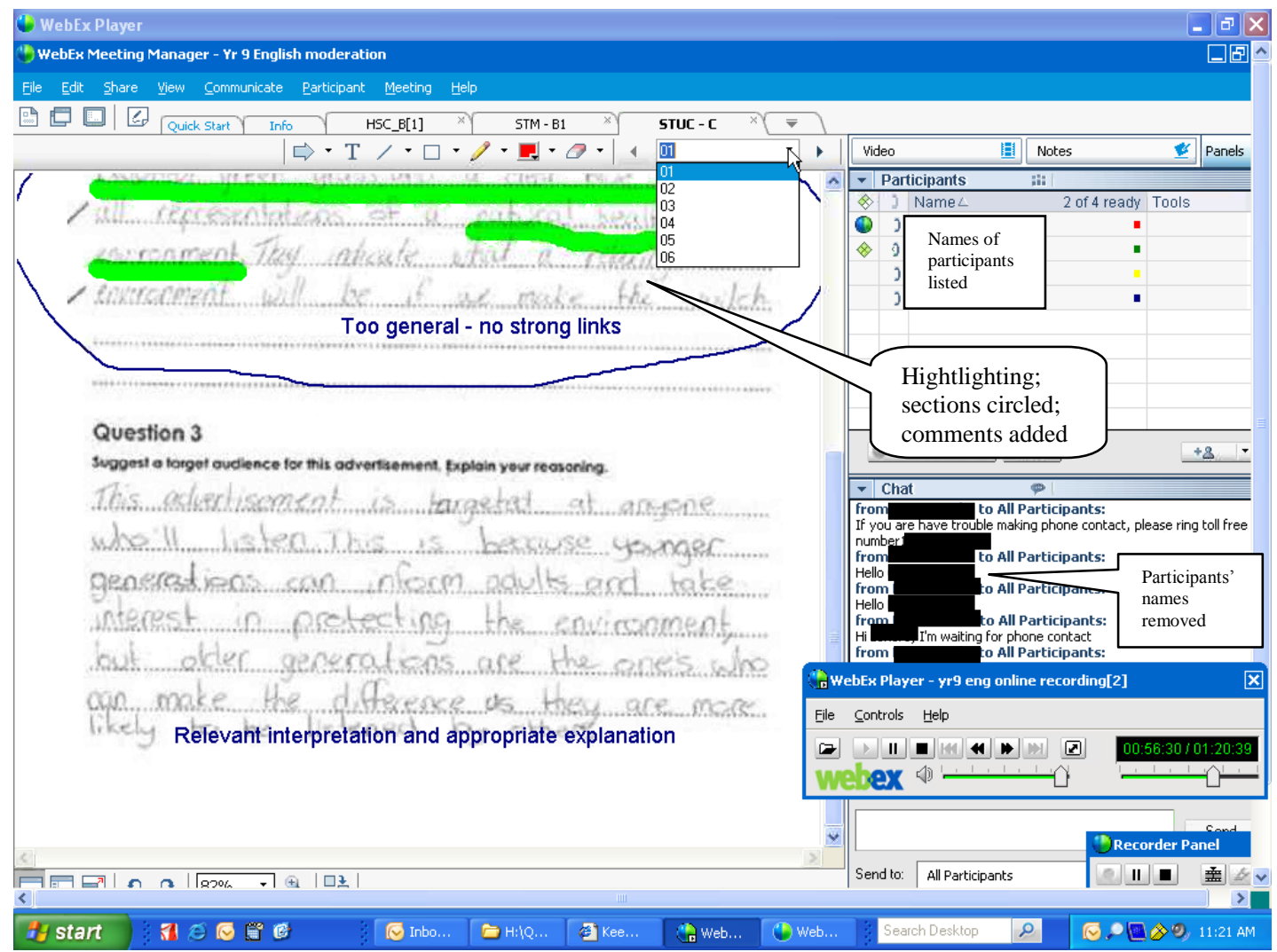

Figure 1: Screen capture of $\mathrm{WebEx} \odot$ meeting in progress

\section{Theoretical and methodological framing}

Methodologically, the study draws on theories of learning, in particular, those regarding the development of communities of practice and the theorising of learning as an act of social practice (Lave \&Wenger, 1991; Wenger, 1998). Within theories of learning, assumptions are made about the individual, the social, and the interaction between the individual and the social. The theorising is not intended as abstraction but rather to purposefully link to the concrete relations that occur in everyday life. This work can help in understanding how learning occurs through participation in social experiences, through a process of legitimate peripheral participation. Within this theory, meaning and community are investigated through the local practices that are informed by wider social, cultural, historical and political contexts. The development of identity within a community of practice is relevant to the context of this research as it investigates the impact of the different sociocultural contexts of individuals on the development of shared meaning. This framework provides one way of investigating how shared understandings may develop through participation in common practices.

Work by Slack and Wise (2005) is also drawn upon to incorporate understandings of the impact of meeting and learning online. The concept of technological culture adds a different lens through which to investigate online moderation meetings within a sociocultural perspective of learning. Slack and Wise (2005) use this concept to accentuate the integration of technology with culture. Culture is understood as a "whole way of life" in which technology is included as part of the artefacts involved in the processes that occur (Slack \& Wise, 2005, p. 4). Culture and technology are not considered as two separate entities that impact on each other. To view technology within a concept of culture, or technological culture, redirects questions away from the relationship between technology and culture, and brings the focus on the issues involving the technology as integral to the culture in which it exists. 
Slack and Wise (2005) understand technology as much more than just an object arguing that it also acts as an agent within societies, shaping while being shaped by the culture within which it exists. This requires a re-examination and redefinition of the terms "agent" and "agency." Within this paper, it is emphasised that technology can only be understood through recourse to the culture in which it is embedded. Technology can be interpreted in multiple ways and take on quite different meanings dependent on the cultural context. The concept of agency is used to describe the nature of the connections within a technological culture. The definition of agency in this context differs from the commonly held definition of this term as usually involving humans, requiring acts of intention, and as something that is possessed. Slack and Wise (2005, p. 117) define agency as a process or a relationship that involves participants but do not limit this participation to humans alone. Agency refers to "the ability to bend space, to make something happen" (Slack \& Wise, 2005 , p. 131). This redefinition of agency broadens the concept to include technologies as participants that can be involved in relations of agency. What appears to be a radical definition of agency, on closer inspection, has been qualified to include technologies while not attributing to those technologies human qualities and ways of interacting, or any sense of intention.

In contrast to this position, Wenger's (1998) interpretation of participation focuses on mutual recognition that involves shaping participants' experience of meaning through negotiation, and then acting with purposeful responsibility for the meanings generated. Since computers cannot perform such social actions, Wenger (1998) considers that computers do not participate in a community of practice but rather perform a role in that practice. This stance appears at first to be in stark contrast to the position of technological agency posited by Slack and Wise (2005).

However, these authors also do not attribute the computer with abilities to respond to engagement. That is not their argument. Rather their view is to understand the computer through the social and cultural context which has given a certain meaning to how it is perceived and used, and the power attributed to it in this role. Furthermore, Slack and Wise (2005) acknowledge the contribution of the computer to forming and shaping identity within a community of practice.

Instead of viewing the computer as an object that performs a function in a community, in this paper, the position of Slack and Wise (2005) is adopted. Wenger's (1998) view of the computer as incapable of engaging in mutual recognition is not rejected; rather the stance is taken that the computer must be viewed as more than just an object that functions within a sociocultural context. When technologies are viewed as agents in everyday life, investigations are opened up to consider the part played by the technology in transforming or contributing to an outcome. For example, teachers involved in ICT-mediated moderation may receive a weak connection and keep dropping out of the meeting thereby reducing their contributions and providing a negative impression of meeting in such an environment; or the dynamics of turn taking in such an environment may cause frustration and inhibit the natural flow of the conversation. Such factors relating to technology play a part in shaping the conversation that will take place. The role of the technology cannot be negated in the dynamics of this context.

In this paper, the computer is understood through the meanings that have been attributed to it through the sociocultural environment in which it is a part of a community of practice. The computer cannot be understood outside of the context in which it is developed and used; the computer is part of the connections which together form the culture (Slack \& Wise, 2005).

\section{Data collection methods and participants}

The participants in this study were 24 teachers from Years 4, 6 and 9 who were involved in online moderation meetings as a way to obtain consensus on the standards awarded for given samples of student work. As the teachers participated in ICT-mediated moderation meetings, their conversations were tape recorded. The analysis in this paper is based on observations of six online moderation meetings, transcripts from two of the online meetings, thirteen pre- and postmoderation interviews, twelve surveys completed by teachers after their involvement in the online meetings, and over two hundred email communications that occurred between the researcher and the participants in the organisation of the meetings. 
The online moderation sessions have been analysed for the type of contributions made by the teachers, and the interactions that occurred between participants. This data has been used to document the social context of group moderation and the factors that have supported or hindered teachers in their progress towards developing a common understanding of the standards in a synchronous online moderation environment. In particular, the focus of this analysis is on the relationship between the agency of the technology and the development of an assessment identity for teachers within a standards-referenced system of assessment. The data collected through observing and recording moderation sessions have been triangulated with the data collected through the interviews, surveys and emails.

\section{Findings}

Lave and Wenger (1991, p. 53) stated that "activities, tasks, functions, and understandings do not exist in isolation; they are part of broader systems of relations in which they have meaning" (p. 53). In this analysis, it is not assumed that the learning that occurs regarding teacher assessment practice is a direct result of teachers' involvement in the online moderation meetings, but rather that the moderation meetings contribute to these changes. Many of the teachers have been involved in other forms of moderation meetings, which have also impacted on their understandings. Likewise, the many discussions that the teachers have had within their work environment and in other contexts have also contributed to their learning. Taking this understanding into consideration, these findings consider the factors that have supported or hindered the teachers working in a synchronous online mode of moderation as they endeavour to develop a shared understanding of a standard of work, while acknowledging that many other factors also contribute to this learning. The following discussion considers these factors in terms of (a) the organisational and technical issues, and (b) the communication issues which emerged from the study. These categories have been established for ease of discussion, but their interconnectedness is evident as the technology either supports or inhibits communication.

The findings to date are optimistic for the developing use of this technology for teachers. An emergent finding from the surveys and post-moderation interviews is that teachers valued the opportunity to talk with others outside of their own school, cluster or region and rated the online moderation process as beneficial. However, many issues still need to be countered and addressed, as the following discussion illustrates.

\section{Organisational and technical issues}

This section considers the problems and solutions the researcher and the participants faced when using this technology. It ranges from issues with sending the tasks for viewing in the online meetings, to entering the meetings and engaging with the online tools.

For each online meeting, the teachers selected the range of samples that would be considered. For example, some groups chose to view an A - E sample from each school, while others moderated a selection from within this range of standards. The teachers were required to send a copy of the selected samples to the researcher so that the samples could be scanned into the computer for the meeting. The teachers had the choice of scanning and emailing samples, photocopying and posting samples, or posting the original samples. For face-to-face moderation meetings, teachers are required to photocopy their samples. Online moderation entailed an extra step of posting or emailing the samples. For some teachers, the time involved in organising student samples for online moderation was an issue. This is exemplified in the following teacher's email to the author (coded as R (Researcher)). 
Hi R

I have been away from school, so I have organised today to have the samples scanned in to the computer and will email them to each person. I should have this done by the end of the day. You can post the samples - without mine, as I will email everyone, if this is appropriate to do so (it will save postponing things). I have to go to class now, but let me know if you have any concerns.

(personal communication, 31/10/2008)

The organisation of assessment tasks for a moderation meeting added another task for this teacher to complete. In this email the teacher explains that she has been away from school but plans to have the samples scanned by the end of the day. This did not occur as planned in the email and later conversations highlighted the many other tasks that needed to be completed as well as teaching duties. Organisational issues such as this are one reason teachers gave for withdrawing their involvement in the online meetings.

Paradoxically, this organisational issue for online moderation is also viewed as one of its strengths. Because teachers receive the student samples before the meeting, they can peruse them in their own time before moderating the work online. The result is an efficient meeting which only involves discussion of the samples, and not the added time of teachers making judgements on unsighted work and needing time to look over each sample. The issue for this form of organisation is that the teachers are required to have their samples sent to the organiser in time for them to be scanned, put into level folders and redistributed to all participants with sufficient time for perusal of all tasks before the meeting. This was an organisational and time issue for many busy teachers.

Many teachers chose to bypass all technology and time issues and post the original samples, while other teachers were willing to attempt scanning and emailing samples. Teacher confidence with using the technology was an important factor as the following emails demonstrate.

\section{Hi R}

I have scanned the 3 samples. I will send them in 3 emails so as not to stress out the server here!!! If they are not clear enough let me know and I can express post them this afternoon. They are images and I think if they are pasted into a document they should be ok to read. Hope this is $O K$.

(personal communication, 30/10/2008)

This teacher evidently has experience with the technology and knows what to do to not overload the school email system. However, some teachers' knowledge regarding the use of this technology limited their attempts. The following two extracts from emails between participants and the researcher illustrate this point.

$R$,

I'm having trouble sending you the scanned student samples by email. Do you have a fax number that I could fax them to on Monday?

(personal communication, 24/10/2008)

Teacher: I can't email my samples! It is saying that the file is too big. We haven't got an ICT person at the school at the moment. Do you know how to make the files smaller?

Researcher: Have you tried individually zipping each file and sending one by one?

Teacher: No, how do I do that? 
In the first example, there was no further attempt to problem solve - the samples were faxed through. In the second example, the samples were faxed the day before the meeting.

The issues raised by these extracts led to another challenge faced when emailing these documents. The tasks were documents that contained tables, pictures and diagrams, and approximately fifteen pages long. These were scanned into the computer as pdf files at a 600 resolution. Scanning at a lower resolution produced a document that was unclear and difficult to read. The file capacity for most school and institution emails could accept these documents as pdfs when they were zipped and sent individually. However, some schools had difficulty opening the documents in a pdf format. Further, the $\mathrm{WebEx}^{\odot}$ online meeting centre does not work with pdf files. So the documents needed to be converted to word documents. This led to the next problem. Converting directly from a pdf file containing pictures, tables and diagrams into a word document produced a very large file even when extraneous sections were cut from the documents. Zipping the file did little to help reduce the size because images cannot be compressed effectively. As a result, the zipped files were still too large to be transmitted from the researcher's email repository. So for teachers who required the documents in Word format, another solution needed to be found. The current solution involves capturing a screen image of the pdf page, pasting this to a Word document and cropping then resizing the image. This is a time-consuming process but has produced to date the clearest image and the smallest file size that all schools are able to receive.

Another difficulty faced by some participants occurred when emailed samples were difficult to read or some pages were missing. This occurred even though all teachers received the same samples in a group email. In another incident, a teacher phoned the day before the meeting enquiring why she had not received copies of the student tasks. The tasks had been sent two days before the request as a group email which other participants had received. Upon request, the tasks were resent to the same email address, in the same format, and received. There are many factors that contribute to the successful sending and receiving of the documents by email including the infrastructure of the many institutions involved in these meetings.

In preparation for one of the online moderation meetings, a teacher had typed the student scripts verbatim because of the poor quality of the scanned samples. While this task was time consuming for the teacher, it also produced uncertainty for the teachers moderating the student samples from this school, as the following extract from the online meeting demonstrates.

Teacher1: $\quad$ Can I just ask a question from [school]. We gave them a higher mark for the editing section of the rubric because we weren't sure, did the students type it up on the computer after the test?...or...um... how did that work? Because we just [photo]copied ours, did the students just look at their actual test and copy it straight from there...or...?

Teacher 2: $\quad$ No, the only reason it appears in type form is that it wouldn't scan clearly enough, for scanning...So I actually typed it word for word and spelling for spelling ... however what I couldn't type in I just said would be editing marks for the children.

Teacher 1: $\quad$ Ok, no that's good. I just wanted to check because I wasn't sure about things like the spelling and the punctuation if they would be changed because it was on the computer. So that's good.

(transcript online moderation meeting, June, 2008)

While the issue appeared resolved for the teachers involved in this meeting, it does raise important ethical issues for this practice. For example, is there a need for teachers to declare that they have typed students' texts and include their reasons for doing so? Further, should the original work samples be sent with typed texts for verification? 
Once all participants had received files that contained legible student documents ahead of the meeting, the next challenge was to connect all participants to the meeting. Of the thirteen meetings that have been organised, only one meeting has failed to run due to a participant not being able to connect. Teachers received the official $\mathrm{WebEx}^{\odot}$ invitation that contained the link to connect to the meeting one or two days prior to each meeting. Teachers were required to click on this link which then led them through the process to enter the meeting. However, things did not always work as planned as can be noted in the following extract,

Researcher: Did you connect through the invitation?

Teacher: $\quad$ No we tried to when it said that ...they said they had a glitch, WebEx ${ }^{\odot}$ itself had a glitch and they logged the problem and would get back to us.

(transcript online moderation meeting, June, 2008)

In the majority of meetings, teachers connected to the meeting through the email link but still some failed to make telephone contact.

Teacher: $\quad$ I wasn't able to... the phone number that I was...the line to the meeting didn't seem to be working... So it took me a long time to get on board.

(post-moderation interview, 31/10/2007)

One way of assisting teachers to overcome this difficulty was to post a message that all participants could see as they entered the meeting:

From [Researcher] to all participants: If you are having trouble making phone contact, please ring toll free number [number]

This decreased the problem teachers experienced making phone contact, and all participants were able to join these meetings. Although this phone number was also included in the information sheets sent to the participants, and in the emailed meeting invitation, having the contact number on the screen of the meeting as the teachers entered, appears to be the most supportive positioning.

Another issue was the time lapse between when the first and last participants entered the meeting. This time lapse can delay the start of the meeting for fifteen to twenty minutes. These meetings are meant to be fiscally and temporally efficient for teachers, so the organisation of, and expectations for participants in these meetings need to be clearly stated. In this case, it is imperative for all participants to promptly join the meetings. The degree of coordination and organisation that is currently required to ensure the running of these meetings is evidenced by the number of emails sent and received as referred to earlier in the paper.

It is apparent that the technological issues are strongly linked to the identity that the participant has formed with the technology. For instance, this participant's email illustrates a certain degree of confidence with the technology. File size is stated, and if there are any difficulties for the other participants then changes can be made.

The files are a little over $2 \mathrm{MB}$... I hope they don't cause problems for anyone. Let me know if they do, I'll make some changes...Seems I will need to send them separately, so 2 more emails follow. 
The relationship between the technology and this participant, in contrast to the relationship for the teacher who appeared to be in a panic about reducing file size and gave up in her attempt to do so is significant. The connection between technology and identity, like all other relationships, will evolve with time. The initial apprehension and uncertainty in working with technology may dissipate as procedures are learnt and teachers start to problem solve based on prior experience. At issue currently, is whether teachers will be deterred by their first experiences to the extent that they are not interested in learning more about this mode of communication. It is a concern, not only because teachers may be excluding themselves from valuable opportunities to learn from others, but also because they are reducing their opportunities to engage in a mode of communication that is increasingly becoming a part of their students' future life and work experiences. The following section focuses on how the teachers worked with this technology to communicate in the meetings.

\section{Communication issues}

From a sociocultural perspective, identity is formed and learning occurs through interactions within a community. Once this community moves into the context of an online meeting and hence out of sight, the role of verbal communication becomes essential. Through the survey responses, participants reported feeling offended by comments made in a meeting which they have attributed to others not being able to see their facial or body language. If participants cannot be heard, if through their text they appear, for example, aggressive or disinterested, then there are no visual cues in the online meetings being conducted to allay these perceptions. Yet, within all survey responses, only one teacher stated that online communication was not easy.

In other meetings participants reported successful attempts to communicate. For example, in a post-moderation interview, one participant stated:

The conversation was clear, despite the fact that you obviously weren't dealing with someone face-to-face it was, I thought it went quite, quite smoothly.

(post-moderation interview, 31/10/2007)

For other participants, reception had been poor with voices frequently breaking up and much background buzz. Although the technical issues of online communications were frequently listed as a hindrance for this form of communication, there were a minimum number of interactions in any of the meetings where participants indicated that they had not heard what another participant had said. More frequently teachers appeared to grasp the main message being articulated and made attempts to respond appropriately. However, one participant did withdraw from a meeting early, later communicating in an email the frustration that was felt due to not being able to hear another participant clearly. This participant had joined the meeting through a mobile phone which had very poor reception.

The inability to see other participants was stated as a problem for many teachers particularly in connection with more than one participant attempting to speak at the same time. Although $\mathrm{WebEx}^{\odot}$ has a hands-up icon that participants can show when they want to speak, it was rarely used in meetings. This is the first, or in limited cases, the second experience that these teachers have had with moderating in an online environment (see Figure 1). From the teachers' responses, it appears evident that online modes of moderation will need to incorporate new protocols for interacting.

The annotation tools that are a part of the $\mathrm{WebEx}^{\odot}$ program proved to be an aid for teachers communicating their understanding of the standards. The teachers used the tools to highlight, and comment on, the evidence in a piece of student work that they believed illustrated a particular standard. 
Teacher 1: $\quad$ I agree that her orientation may not be as complex as it could be but she has used good words as you have highlighted ...

Teacher 2: $\quad$ Oh good, colouring-in I like it!

(transcript online moderation meeting, June, 2008)

The tools directly contributed to teachers focussing on the evidence provided in the samples. Yet, in many cases observed, the teachers, while acknowledging another's perspective, still did not move towards accepting this reasoning. Often a difference in opinion was attributed to a difference in teaching focus or emphasis, a different interpretation of what constitutes a pass standard (C or $\mathrm{D}$ ?), or the shortcomings of the task and criteria sheet.

Another interesting facet of the meetings was the communicative role of 'key' participants. These participants could be identified through their interactions that contributed to the functioning of the meeting. In these meetings key participants acknowledged, responded to, and initiated conversations almost as many times as the researcher. Contributions that acknowledge another's statements may consist of a simple utterance, for example, "yes", "ok", "uhah"; or a phrase such as, "ok, fair enough", "thank you for that", "Yeah, I hear what you are saying"; or through the repetition of a phrase used by the previous speaker. Key participants continued in this role throughout the meeting by interacting far more than any other participant whether agreeing, disagreeing, or questioning others. The role of the key participants was important to the running of the meeting, and also as a model of how to operate within this context. However, when key participants dominated the conversation and acted as a voice of authority, the negotiation process was inhibited.

In an online environment, it is easy for dominant participants to take over while others are silenced. For example, in one meeting, a younger teacher interacted only when invited and did not raise any disagreements with judgements made by others. The limited comments made by this participant were presented in such an indefinite manner that could be viewed as supportive of any position. This peripheral involvement in a meeting suggests an assessment identity that is not fully developed. The participant was hesitant to contribute to the meeting and was more involved with 'observing' interactions and negotiations of the experienced teachers rather than directly participating. Lave and Wenger (1991) believe that the skill to perform in a particular context is acquired through increasingly sophisticated opportunities to engage in the activities and knowledge of a community. Knowledge and skills are developed through the exposure of perspectives and the negotiation of meanings so that transformation occurs most radically to someone new to the community. For this young teacher, it could be anticipated (but not guaranteed) that her future involvement in moderation practices, including online opportunities, would result in increased involvement and contribution to the meetings.

\section{Discussion}

Identity is built through interactions with other participants in the online meetings, and further constructed and reconstructed through the other interconnected networks which constitute the sociocultural environment. Technology is not a neutral object in this context (Slack \& Wise, 2005). The success of teachers to interact in this environment is linked in some manner to their relationship with the technology. Teachers do not enter these meetings equally.

The relationship between a "technological" identity and having the confidence to moderate online appears to be significant for the sample of teachers in this study. Teachers identify themselves as being technologically 'savvy' or not. This can be noted in the following comment: 
I guess that I don't consider myself to be the most savvy computer person, but I found it okay... I'm just not sure how a lot of other teachers would find it... I'm just not sure how it will work on a large scale.

(post-moderation interview, 19/10/2007)

Teachers' vulnerability to such a process is evident in their comments and their uncertainty as to how this process may progress on a large scale, and how it may work for themselves and others. The teachers' identities shape, and are shaped by, the technological culture in which they must operate (Slack \& Wise, 2005). While the teachers have chosen to be a part of this culture, they have also chosen to accept, or to resist certain aspects of the culture. For example, while the teacher (above extract), positioned himself on the boundaries of this culture, he also positioned others as lacking in the expertise to participate in such practices, and hence questioned the development of such an assessment culture. The introduction of such a culture into an education system challenges notions of identity as teachers position themselves as technologically 'savvy' or not. As these tensions are resolved, as new experiences become common practice, then transformation in terms of identity may occur (Stevenson, 2008).

Lave and Wenger stated that "the social relations of apprentices within a community change through their direct involvement in activities" (p. 94), in which "understanding and knowledgeable skills develop". This understanding of learning through apprenticeship involves more than just observing from the sidelines, learning involves participating in increasingly, complex activities that are a part of a community of practice. In the online social moderation meetings teachers' involvement is indicative of their confidence to work in such an environment and with themselves as an assessor who can work within a standards-based system. This work requires that teachers can competently explicate, justify and negotiate their understanding of standards. Teachers' involvement in the moderation meetings is reflective of the roles (new-comers and experts) that they have taken up within the moderation practice.

To develop a shared meaning of a standard requires that participants are willing to not only acknowledge new perspectives but to also value their contribution to forming a consistent judgement. The confidence gained by teachers as a result of their judgements being agreed on by someone outside of the school or cluster is significant. Eraut (2008) has identified the importance of confidence in learning, and the role that this plays in the openness of teachers to opportunities for learning and their willingness to address challenges. The opportunity to participate in the online moderation with teachers from another district appears to support the development of an assessment identity as one who is a competent judge of student work within a standards-referenced system. The enthusiasm of the teachers to learn how others judged student work and whether the standards were being interpreted in a similar way is evident in this teacher's response.

The external moderation was excellent. I thought the process worked really, really well and, as I said, we reached consensus on most of the samples that we were viewing.

(post-moderation interview, 31/10/2007)

Online moderation provides teachers with the opportunity to develop as competent assessors within a standards-based assessment system by having their judgement process validated outside of their local geographical boundaries. At the same time, teachers need to be open to, and feel supported in, these opportunities for learning. 


\section{Conclusion}

The tension between the processes that support or hinder teachers in developing shared understanding of standards is evident as teachers welcome the opportunity to talk with peers across the state yet struggle with using the technology. Learning to work within an online environment to conduct moderation meetings requires that teachers become familiar with new tools and social protocols to support this form of communication.

If assessment is to play an integral role in the promotion of student learning, then practice at the local level of the classroom needs to be shared, justified and negotiated on a much wider scale. Isolated understandings do not lead to a strong, unified organisation, particularly when variations in quality exist. While online modes of moderation present such an opportunity, there are many obstacles still to overcome. This paper has highlighted some of the technical and communication issues that have supported but mainly hindered teachers in their attempts to operate within such an environment. However, when reflecting on the hindrances to this form of communication, all of the obstacles can be overcome. The developments that are being made with this technology will over time work to reduce some of the current problems, such as connection to meetings. Further, as teachers continue to use the technology it is anticipated that their confidence should increase, supporting their agency to operate in such a culture, thereby resulting in a transformation of identity within this assessment culture.

The factors discussed in this paper have relevance for teachers developing identities as competent assessors of student work. Further investigations of this topic will focus on whether, and how, the learning that occurs as teachers are involved in online moderation meetings may be generalised to their teaching context. It is imperative that as a new curriculum, assessment and reporting policy is introduced for teachers that understandings are developed of how this policy is being enacted and experienced by teachers in the online moderation meetings, and as this is translated to the classroom context.

\section{References}

Davidson, J. (1999). All things in moderation: A whole school approach to authentic assessment. In H. Fehring (Ed.), Literacy assessment: A collection of articles from the Australian Literacy Educators' Association (pp. 81-84). Newark, USA: International Reading Association.

Department of Education Training and the Arts. (2005). Queensland curriculum, assessment and reporting. Brisbane, Australia: Strategic Policy and Education Futures (Department of Education and the Arts, Queensland Government).

Department of the Premier and Cabinet. (2005). Smart Queensland: Smart State strategy 2005 2015. Brisbane, Australia: Queensland Government.

Eraut, M. (2008). Learning from other people in the workforce. In K. Hall, P. Murphy \& J. Soler (Eds.), Pedagogy and practice: Culture and identities (pp. 40 - 57). Milton Keynes, UK: The Open University.

Fehring, H. (1998). Understanding the influences of teachers' judgements in the process of assessing and reporting students' literacy in the classroom. Paper presented at the Literacy for All: Joint National Conference Australian Literacy Educators' Association \& Australian Association for the Teaching of English.

Ingvarson, L. (1990). Enhancing professional skill and accountability in the assessment of student learning. Paper presented at the Annual Meeting of the American Educational Research Association.

Klenowski, V. (2007). Evaluation of the effectiveness of the consensus-based standards validation process [Electronic Version]. Retrieved January 14, 2008, from http://education.qld.gov.au/corporate/newbasics/html/lce_eval.html 
Lave, J., \& Wenger, E. (1991). Situated learning: Legitimate peripheral participation. Cambridge, UK: Cambridge University Press.

Malone, L., Long, K., \& De Lucchi, L. (2004). All things in moderation. Science and Children, 41(5), 30-34.

Maxwell, G. (2002). Moderation of teacher judgements in student assessment: Discussion paper on assessment and reporting. Brisbane, Australia: Queensland School Curriculum Council.

Queensland Studies Authority. (2005). Moderation processes for senior certification. Brisbane, Australia: Queensland Studies Authority.

Queensland Studies Authority. (2007). Information statement February 2007: Essential learnings draft 2. Brisbane, Australia: Queensland Studies Authority.

Salmon, G. (2003). E-moderating : The key to teaching and learning online. ( $2^{\text {nd }}$ ed.). London, UK: RoutledgeFalmer.

Slack, J. D., \& Wise, J. M. (2005). Culture + technology: A primer. New York, USA: Peter Lang Publishing.

Stevenson, J. (2008). Concepts of workplace knowledge. In P. Murphy \& R. McCormick (Eds.), Knowledge and practice: Representations and identities (pp. 46 - 57). Milton Keynes, UK: The Open University.

Wenger, E. (1998). Communities of practice: Learning, meaning and identity. Cambridge, U.K: Cambridge University Press.

Copyright (C) 2010 Lenore Adie 